\title{
ITERATIVE GRADIENT TECHNIQUE FOR THE DESIGN OF LEAST SQUARES OPTIMAL FIR MAGNITUDE SQUARED NYQUIST FILTERS
}

\author{
Andre Tkacenko and P. P. Vaidyanathan \\ Dept. of Electrical Engineering \\ California Institute of Technology, Pasadena, CA 91125, USA \\ E-mail: andre@systems.caltech.edu,ppvnath@systems.caltech.edu
}

\begin{abstract}
Recently, much attention has been given to the design of optimal finite impulse response (FIR) compaction filters. Such filters, which arise in the design of optimal signal-adapted orthonormal FIR filter banks, satisfy a magnitude squared Nyquist constraint in addition to the inherent FIR assumption. In this paper, we focus on the least squares optimal design of FIR filters whose magnitude squared response satisfies a Nyquist constraint. Using a complete characterization of such systems in terms of Householder-like building blocks, an iterative gradient based greedy algorithm is proposed to design such filters. Simulation results provided show the merit of the proposed technique for designing FIR compaction filters. ${ }^{1}$
\end{abstract}

\section{INTRODUCTION}

In recent years, much attention has been given to the design of signal-adapted orthonormal filter banks $[5,8,1]$, which find applications in data compression. The typical signal model in this setting is the $M$-channel uniform maximally decimated filter bank shown in Fig. 1 [7]. Here, the input $x(n)$ is assumed to be cyclo wide sense stationary of period $M(\operatorname{CWSS}(M))$ [7], which is tantamount to saying that its $M$-fold blocked version $\mathbf{x}(n)$ is wide sense stationary (WSS) with some power spectral density (psd) $\mathbf{S}_{\mathbf{x x}}(z)$. If the analysis/synthesis filters of Fig. 1 are constrained to satisfy an orthonormality condition [7] but are of unconstrained order, then the optimal filter bank for a number of objectives happens to be an infinite order principal component filter bank (PCFB) for the psd $\mathbf{S}_{\mathbf{x x}}(z)[8,1]$. Such filter banks are simultaneously optimal for several relevant data compression objectives such as coding gain and multiresolution. The optimal analysis/synthesis filters in this case turn out to be a series of compaction filters [5, 8]. In many practical cases of inputs (for example, if $x(n)$ is itself WSS), these filters are ideal bandpass filters [8] and hence unrealizable.

If we restrict our attention to finite impulse response (FIR) analysis/synthesis filters, then the design of such optimal filter banks becomes far more difficult, since a PCFB in general does not exist [1]. As such, the filters must be designed to optimize a specific objective. In [10], the authors considered maximizing the coding gain, whereas in [3], the authors focused on optimizing a multiresolution criterion. For both methods, numerical optimization techniques must be employed which are not guaranteed to reach a globally optimal solution. However, in [3], it was shown that for the multiresolution criterion considered, the design problem could be greatly simplified since the entire filter bank can be constructed by designing a single FIR compaction filter, followed

\footnotetext{
${ }^{1}$ Work supported in part by the ONR grant N00014-99-1-1002, USA.
}

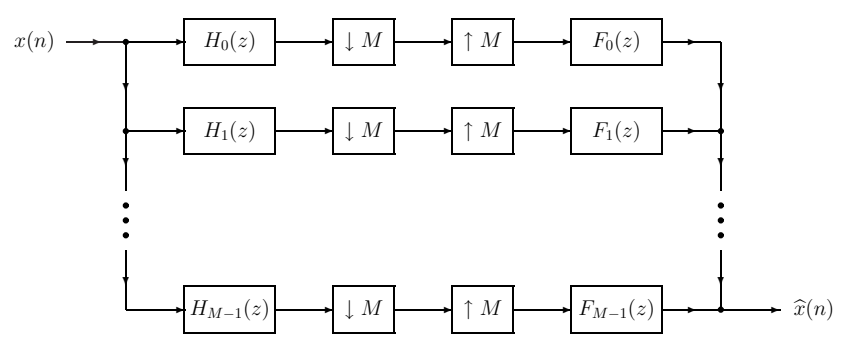

Fig. 1. Uniform maximally decimated $M$-channel filter bank.

by an appropriate Karhunen-Loève transform (KLT). This is possible due to the complete parameterization of FIR paraunitary systems in terms of Householder-like building blocks as shown in [9].

As such, much attention in the signal processing community has focused on the design of FIR compaction filters [3, 2, 6]. In addition to the FIR constraint inherent on such filters, they also satisfy the condition that their magnitude squared response satisifies the Nyquist $(M)$ property [7]. The authors in [3] proposed a linear semi-infinite programming (SIP) method for the design of such filters. Though their method is globally optimal for the problem considered, the resulting magnitude squared response only satisfies the Nyquist $(M)$ constraint on a discrete set of frequencies. Furthermore, their method becomes overly computationally complex as the filter order increases. In [2], a window method for the design of FIR compaction filters was proposed. Though this method is not prohibitively computationally complex for large filter orders, it is inherently suboptimal. In [6], the authors proposed a method for the design of a globally optimal FIR compaction filter based on an appropriate state-space decomposition of the magnitude squared response. Though this method is globally optimal, it only applies for the special case in which the input signal $x(n)$ is WSS and, like the SIP method, becomes very computationally intensive as the filter order increases.

In this paper, we focus on the least squares design of FIR filters whose magnitude squared response is constrained to satisfy the Nyquist $(M)$ property. Using the complete Householder-like factorization of such systems from [9], an iterative algorithm is proposed for the design of such filters. The proposed technique is a greedy algorithm in that the observed mean squared error is always monotonic nonincreasing as a function of iteration. Furthermore, it can be applied for a wide variety of design problems and is computationally inexpensive as it only involves the numerical computation of integrals and eigenvectors. Simulation results provided show that this method can yield FIR compaction filters that are very close to the infinite order ideal bandpass compaction filters. 


\section{LEAST SQUARES DESIGN OF FIR MAGNITUDE SQUARED NYQUIST FILTERS}

The design problem we focus on here is as follows. Suppose that $D\left(e^{j \omega}\right)$ is a desired response that we wish to approximate (in the least squares sense) with a causal FIR filter $F\left(e^{j \omega}\right)$ of length $M N$ whose magnitude squared response is $\operatorname{Nyquist}(M)$ [7]. Then, the problem is to minimize the mean squared error $\xi$, defined below,

$$
\xi \triangleq \frac{1}{2 \pi} \int_{0}^{2 \pi}\left|D\left(e^{j \omega}\right)-F\left(e^{j \omega}\right)\right|^{2} d \omega
$$

subject to the FIR constraint on $F\left(e^{j \omega}\right)$, as well as the constraint,

$$
\left[\left|F\left(e^{j \omega}\right)\right|^{2}\right]_{\downarrow M}=1
$$

Expanding $\xi$ from (1) yields,

$$
\begin{aligned}
\xi & =\frac{1}{2 \pi} \int_{0}^{2 \pi}\left|D\left(e^{j \omega}\right)\right|^{2} d \omega+\frac{1}{2 \pi} \int_{0}^{2 \pi}\left|F\left(e^{j \omega}\right)\right|^{2} d \omega \\
& -\frac{1}{2 \pi} \int_{0}^{2 \pi}\left(D^{*}\left(e^{j \omega}\right) F\left(e^{j \omega}\right)+F^{*}\left(e^{j \omega}\right) D\left(e^{j \omega}\right)\right) d \omega
\end{aligned}
$$

Note that the first term of the right hand side in (3) is just the $\ell_{2}$ norm squared of the desired impulse response $d(n)$, namely $\|d(n)\|_{2}^{2}$. Also, using (2), it can be shown that the second term in (3) is simply unity [7]. Hence, the only quantities in $\xi$ which depend on the filter $F(z)$ are the cross product terms appearing in the third term in (3). This greatly simplifies the optimization problem.

From Vaidyanathan et al. [9], it is known that $F(z)$ must be of the form,

$$
F(z)=\widetilde{\mathbf{a}}(z) \mathbf{V}\left(z^{M}\right) \mathbf{u}_{0}
$$

Here $\mathbf{a}(z)$ is the $M \times 1$ advance chain vector given by,

$$
\mathbf{a}(z)=\left[\begin{array}{llll}
1 & z & \cdots & z^{M-1}
\end{array}\right]^{T}
$$

(For the tilde notation [7], recall that $\widetilde{\mathbf{A}}(z) \triangleq \mathbf{A}^{\dagger}\left(1 / z^{*}\right)$ for any $\mathbf{A}(z)$.) The quantity $\mathbf{V}(z)$ is an $M \times M$ lossless matrix consisting of $(N-1)$ Householder-like paraunitary degree-one building blocks of the form,

$$
\begin{gathered}
\mathbf{V}(z)=\prod_{i=N-1}^{1} \mathbf{V}_{i}(z) \\
\mathbf{V}_{i}(z)=\mathbf{I}-\mathbf{v}_{i} \mathbf{v}_{i}^{\dagger}+z^{-1} \mathbf{v}_{i} \mathbf{v}_{i}^{\dagger}, 1 \leq i \leq N-1
\end{gathered}
$$

where the $M \times 1$ vectors $\mathbf{v}_{i}$ are unit norm vectors. Finally, the quantity $\mathbf{u}_{0}$ is some $M \times 1$ unit norm vector.

As can be seen, $F(z)$ in this case is completely characterized by the unit norm vectors $\mathbf{v}_{i}$ and $\mathbf{u}_{0}$. Though it is difficult to jointly find the vectors $\mathbf{v}_{i}$ and $\mathbf{u}_{0}$ which minimize $\xi$ from (1), it will be shown that given the rest of the vectors, optimizing only one vector at a time is very simple. This will serve as the basis for our proposed iterative technique. Prior to proceeding, let us define $\mathcal{V}$ to be the set of all $\mathbf{v}_{i}$ s, i.e. $\mathcal{V} \triangleq\left\{\mathbf{v}_{i}: 1 \leq i \leq N-1\right\}$ and, in accordance with standard set theoretic notation, let $\mathcal{V} / \mathbf{v}_{k}$ denote the set $\mathcal{V}$ with the element $\mathbf{v}_{k}$ removed.

\section{SOLVING THE LEAST SQUARES OPTIMIZATION PROBLEM USING LAGRANGE MULTIPLIERS}

In order to minimize $\xi$ from (3) subject to the unit norm constraints on $\mathbf{u}_{0}$ and the $\mathbf{v}_{i}$ s, we construct the Lagrangian [4],

$$
J\left(\mathbf{u}_{0}, \mathcal{V}\right) \triangleq \xi\left(\mathbf{u}_{0}, \mathcal{V}\right)+\lambda_{0}\left(1-\mathbf{u}_{0}^{\dagger} \mathbf{u}_{0}\right)+\sum_{i=1}^{N-1} \lambda_{i}\left(1-\mathbf{v}_{i}^{\dagger} \mathbf{v}_{i}\right)
$$

where the $\lambda_{i}$ s are the Lagrange multipliers which are chosen to satisfy the unit norm constraints. In what follows, we will consider the problem of optimizing one vector at a time, assuming that the rest of the vectors are fixed.

\subsection{Optimal choice of $\mathbf{u}_{0}$}

To find the optimal choice of $\mathbf{u}_{0}$, we set the conjugate gradient of $J\left(\mathbf{u}_{0}, \mathcal{V}\right)$ with respect to $\mathbf{u}_{0}$ to be zero. From (7), we have [4],

$$
\nabla_{\mathbf{u}_{0}^{\dagger}} J=\nabla_{\mathbf{u}_{0}^{\dagger}} \xi-\lambda_{0} \mathbf{u}_{0}=\mathbf{0}
$$

Using (4) in (3) yields,

$$
\xi\left(\mathbf{u}_{0}, \mathcal{V}\right)=\|d(n)\|_{2}^{2}+1-\mathbf{b}^{\dagger}(\mathcal{V}) \mathbf{u}_{0}-\mathbf{u}_{0}^{\dagger} \mathbf{b}(\mathcal{V})
$$

where $\mathbf{b}(\mathcal{V})$ is the $M \times 1$ vector,

$$
\mathbf{b}(\mathcal{V}) \triangleq \frac{1}{2 \pi} \int_{0}^{2 \pi} \mathbf{V}^{\dagger}\left(e^{j \omega M}\right) \mathbf{a}\left(e^{j \omega}\right) D\left(e^{j \omega}\right) d \omega
$$

Differentiating $\xi$ from (9) with respect to $\mathbf{u}_{0}^{\dagger}$ yields [4],

$$
\nabla_{\mathbf{u}_{0}^{\dagger}} \xi=-\mathbf{b}(\mathcal{V})
$$

Substituting this into (8) yields the optimal choice of $\mathbf{u}_{0}$ given by $\mathbf{u}_{0}=-\frac{1}{\lambda_{0}} \mathbf{b}(\mathcal{V})$. In order to satisfy the unit norm constraint $\mathbf{u}_{0}^{\dagger} \mathbf{u}_{0}=1$, it follows that the optimal $\mathbf{u}_{0}$ is of the form,

$$
\mathbf{u}_{0}=e^{j \alpha}\left(\frac{\mathbf{b}(\mathcal{V})}{\|\mathbf{b}(\mathcal{V})\|}\right)
$$

where $\alpha \in[0,2 \pi)$ is some phase factor. To find the optimal choice of $\alpha$ here, we substitute (11) into (9). This yields,

$$
\xi=\|d(n)\|_{2}^{2}+1-2\|\mathbf{b}(\mathcal{V})\| \cos \alpha
$$

Clearly, to minimize $\xi$, we must choose $\alpha=0$. Hence, the optimal choice of $\mathbf{u}_{0}$ and corresponding $\xi$ are given by the following.

$$
\mathbf{u}_{0, \mathrm{opt}}=\frac{\mathbf{b}(\mathcal{V})}{\|\mathbf{b}(\mathcal{V})\|}, \xi_{\mathrm{opt}}=\|d(n)\|_{2}^{2}+1-2\|\mathbf{b}(\mathcal{V})\|
$$

\subsection{Optimal choice of $\mathbf{v}_{k}$}

In order to find the optimal choice of $\mathbf{v}_{k}$ assuming that all other vectors are fixed, we must cleverly extract only those portions of $\xi$ which depend on $\mathbf{v}_{k}$. For simplicity, let us define the following $M \times M$ matrices.

$$
\mathcal{L}_{k}(z) \triangleq \begin{cases}\prod_{i=N-1}^{k+1} \mathbf{V}_{i}(z), & 0 \leq k \leq N-2 \\ \mathbf{I}, & k=N-1\end{cases}
$$




$$
\mathcal{R}_{k}(z) \triangleq \begin{cases}\mathbf{I}, & k=1 \\ \prod_{i=k-1}^{1} \mathbf{V}_{i}(z), & 2 \leq k \leq N\end{cases}
$$

Note that $\mathcal{L}_{k}(z)$ and $\mathcal{R}_{k}(z)$ are, respectively, the left and right neighbors of the matrix $\mathbf{V}_{k}(z)$ for $1 \leq k \leq N-1$ appearing in $\mathbf{V}(z)$ from (5). In other words, we have,

$$
\mathbf{V}(z)=\mathcal{L}_{k}(z) \mathbf{V}_{k}(z) \mathcal{R}_{k}(z), 1 \leq k \leq N-1
$$

Also note that by construction, we have $\mathcal{L}_{0}(z)=\mathcal{R}_{N}(z)=\mathbf{V}(z)$. Substituting (15) and (6) into (4) and (3) yields,

$$
\xi=\|d(n)\|_{2}^{2}+1-2 \operatorname{Re}\left[c\left(\mathbf{u}_{0}, \mathcal{V} / \mathbf{v}_{k}\right)\right]+\mathbf{v}_{k}^{\dagger} \mathbf{T}\left(\mathbf{u}_{0}, \mathcal{V} / \mathbf{v}_{k}\right) \mathbf{v}_{k}
$$

where the $1 \times 1$ scalar $c\left(\mathbf{u}_{0}, \mathcal{V} / \mathbf{v}_{k}\right)$ and $M \times M$ matrix $\mathbf{T}\left(\mathbf{u}_{0}, \mathcal{V} / \mathbf{v}_{k}\right)$ are defined as follows.

$$
\begin{aligned}
c\left(\mathbf{u}_{0}, \mathcal{V} / \mathbf{v}_{k}\right) \triangleq \frac{1}{2 \pi} \int_{0}^{2 \pi} & D^{*}\left(e^{j \omega}\right) \mathbf{a}^{\dagger}\left(e^{j \omega}\right) \\
& \mathcal{L}_{k}\left(e^{j \omega M}\right) \mathcal{R}_{k}\left(e^{j \omega M}\right) \mathbf{u}_{0} d \omega
\end{aligned}
$$

$\mathbf{T}\left(\mathbf{u}_{0}, \mathcal{V} / \mathbf{v}_{k}\right) \triangleq \mathbf{A}\left(\mathbf{u}_{0}, \mathcal{V} / \mathbf{v}_{k}\right)+\mathbf{A}^{\dagger}\left(\mathbf{u}_{0}, \mathcal{V} / \mathbf{v}_{k}\right)$, where,

$$
\begin{array}{r}
\mathbf{A}\left(\mathbf{u}_{0}, \mathcal{V} / \mathbf{v}_{k}\right) \triangleq \frac{1}{2 \pi} \int_{0}^{2 \pi} \mathcal{R}_{k}\left(e^{j \omega M}\right) \mathbf{u}_{0}\left(1-e^{-j \omega M}\right) \\
D^{*}\left(e^{j \omega}\right) \mathbf{a}^{\dagger}\left(e^{j \omega}\right) \mathcal{L}_{k}\left(e^{j \omega M}\right) d \omega
\end{array}
$$

It should be emphasized that from (17) and (18), the quantities $c\left(\mathbf{u}_{0}, \mathcal{V} / \mathbf{v}_{k}\right)$ and $\mathbf{T}\left(\mathbf{u}_{0}, \mathcal{V} / \mathbf{v}_{k}\right)$ do not depend on the vector $\mathbf{v}_{k}$.

As before, to find the optimal choice of $\mathbf{v}_{k}$, we set the conjugate gradient of the Lagrangian $J\left(\mathbf{u}_{0}, \mathcal{V}\right)$ from (7) with respect to $\mathbf{v}_{k}$ to be the zero vector. From (7), we have [4],

$$
\nabla_{\mathbf{v}_{k}^{\dagger}} J=\nabla_{\mathbf{v}_{k}^{\dagger}} \xi-\lambda_{k} \mathbf{v}_{k}=\mathbf{0}
$$

Differentiating $\xi$ from (16) with respect to $\mathbf{v}_{k}$ yields [4],

$$
\nabla_{\mathbf{v}_{k}^{\dagger}} \xi=\mathbf{T}\left(\mathbf{u}_{0}, \mathcal{V} / \mathbf{v}_{k}\right) \mathbf{v}_{k}
$$

Substituting this into (19) yields,

$$
\mathbf{T}\left(\mathbf{u}_{0}, \mathcal{V} / \mathbf{v}_{k}\right) \mathbf{v}_{k}=\lambda_{k} \mathbf{v}_{k}
$$

which is just an eigenvector equation. In order to minimize $\xi$ from (16), by Rayleigh's principle [4, 7], $\mathbf{v}_{k}$ must be a unit norm eigenvector corresponding to the smallest eigenvalue of $\mathbf{T}\left(\mathbf{u}_{0}, \mathcal{V} / \mathbf{v}_{k}\right)$, which we will denote here by $\mu_{k, \min }$. If $\mathbf{w}_{k, \min }$ denotes any unit norm eigenvector corresponding to $\mu_{k \text {,min }}$, then the optimal $\mathbf{v}_{k}$ and corresponding error $\xi$ are given by the following.

$$
\begin{gathered}
\mathbf{v}_{k, \text { opt }}=\mathbf{w}_{k, \text { min }}, \\
\xi_{\text {opt }}=\|d(n)\|_{2}^{2}+1-2 \operatorname{Re}\left[c\left(\mathbf{u}_{0}, \mathcal{V} / \mathbf{v}_{k}\right)\right]+\mu_{k, \min }
\end{gathered}
$$

To summarize the results of this section, we have shown that using the complete factorization of causal FIR magnitude squared Nyquist $(M)$ systems in terms of Householder-like building blocks, the optimal parameter vectors $\left(\mathbf{u}_{0}\right.$ and the $\left.\mathbf{v}_{i} \mathrm{~s}\right)$ can be easily found one at a time assuming that the rest of the vectors are fixed. This property will form the basis of an iterative algorithm for solving the original least squares problem.

\section{ITERATIVE GRADIENT OPTIMIZATION ALGORITHM}

Let $\xi_{m}$ denote the error at the $m$-th iteration for $m \geq 0$. Then, the iterative gradient optimization algorithm is as follows.

\section{Initialization:}

1. Generate $N$ random unit norm vectors $\mathbf{u}_{0}, \mathbf{v}_{i}, 1 \leq i \leq N-1$.

2. Compute the matrix $\mathcal{R}_{N}(z)$ using (14).

Iteration: For $m \geq 0$, do the following.

1. If $m$ is a multiple of $N$ :

(a) Calculate the optimal vector $\mathbf{u}_{0}$ and corresponding error $\xi_{m}$ using (12) and (10) with $\mathbf{V}(z)=\mathcal{R}_{N}(z)$.

(b) Compute $\mathcal{L}_{0}(z)=\mathbf{V}(z)$ and $\mathcal{R}_{1}(z)=\mathbf{I}$.

Otherwise, if $m \equiv k \bmod N$ where $1 \leq k \leq N-1$ :

(a) From (13), update the left matrix as $\mathcal{L}_{k}(z)=\mathcal{L}_{k-1}(z) \widetilde{\mathbf{V}}_{k}(z)$.

(b) Calculate the optimal vector $\mathbf{v}_{k}$ and corresponding error $\xi_{m}$ using (20), (17), and (18).

(c) From (14), update the right matrix as $\mathcal{R}_{k+1}(z)=\mathbf{V}_{k}(z) \mathcal{R}_{k}(z)$.

2. Increment $m$ by 1 and return to Step 1 .

As the iterations progress, the left matrix is shortened by the old optimal vectors $\mathbf{v}_{k}$ whereas the right matrix is lengthened by the newly computed ones. After all of the vectors $\mathbf{v}_{k}$ have been optimized, the left matrix assumes the value of the right matrix while the right matrix is then refreshed to be the identity matrix.

Since at each stage in the iteration, we are optimizing one vector while fixing the rest, the above technique is a greedy algorithm. As such, the mean squared error $\xi_{m}$ is guaranteed to be monotonic nonincreasing as a function of the iteration index $m$. Furthermore, as $\xi_{m}$ has a lower bound (i.e. we always have $\xi_{m} \geq 0$ ), $\xi_{m}$ is guaranteed to have a limit as $m \rightarrow \infty$ [7]. Simulation results provided here verify this monotonic and limiting behavior as we now show.

\section{SIMULATION RESULTS}

Here, we will consider the design of a compaction filter for a WSS input $x(n)$ with psd $S_{x x}\left(e^{j \omega}\right)$. In this case, the ideal compaction filter magnitude response must satisfy [8],

$$
\left|D\left(e^{j \omega}\right)\right|^{2}= \begin{cases}M, & \omega \in \boldsymbol{\omega}_{x} \\ 0, & \text { otherwise }\end{cases}
$$

where $\omega_{x}$ is the set of frequencies defined as follows.

$\boldsymbol{\omega}_{x} \triangleq\left\{\omega \in[0,2 \pi): S_{x x}\left(e^{j \omega}\right) \geq S_{x x}\left(e^{j\left(\omega+\frac{2 \pi \ell}{M}\right)}\right) \forall 1 \leq \ell \leq M-1\right\}$

For example, if $x(n)$ is a real autoregressive order $4(\mathrm{AR}(4))$ process with psd $S_{x x}\left(e^{j \omega}\right)$ as in Fig. 2, the magnitude squared response of the ideal compaction filter for $M=3$ is as shown in Fig. 2.

To test our proposed method, we considered designing an FIR compaction filter with $N=16$. Though the phase of an ideal compaction filter is arbitrary, we opted for a linear phase spectral factor with a delay equal to half of the FIR filter order. Namely, we chose,

$$
D\left(e^{j \omega}\right)=\left|D\left(e^{j \omega}\right)\right| e^{j \phi(\omega)}, \text { where } \phi(\omega)=\left(\frac{M N-1}{2}\right) \omega
$$

A plot of the observed error $\xi_{m}$ as a function of the iteration index $m$ is shown in Fig. 3 for a total of $K N$ iterations, where we chose $K=\left\lceil\frac{1,000}{N}\right\rceil$. (We opted for an integer multiple of $N$ iterations to ensure that all of the vectors were optimized the same number of times.) As can be seen from Fig. 3, the observed error is indeed monotonic nonincreasing and appears to be approaching a limit. In 


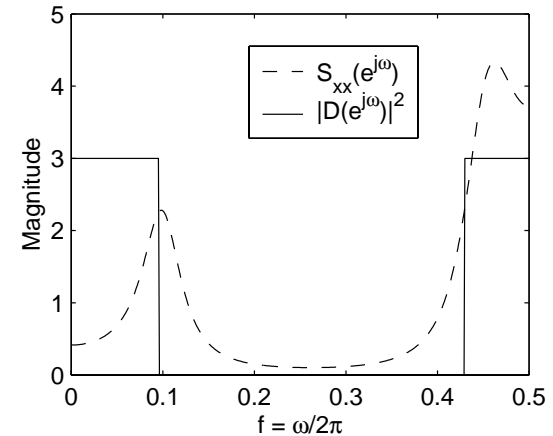

Fig. 2. Input psd $S_{x x}\left(e^{j \omega}\right)$ and ideal compaction filter magnitude squared response $\left|D\left(e^{j \omega}\right)\right|^{2}$ for $M=3$.

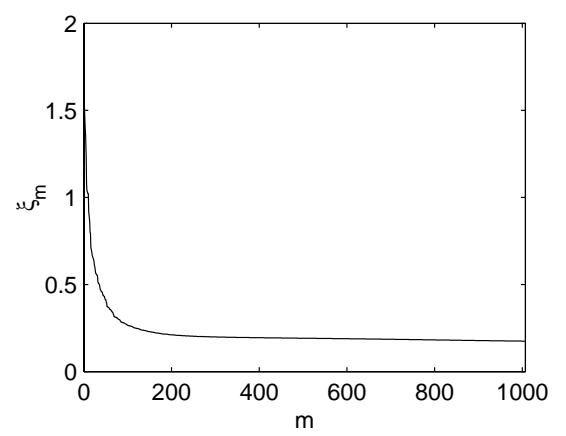

Fig. 3. Mean squared error $\xi_{m}$ vs. the iteration index $m$.

Fig. 4, plots of the magnitude squared responses of the ideal and FIR compaction filters are shown. It can be seen that the FIR filter designed here is a good approximation to the ideal response.

To measure the performance of the our designed FIR compaction filters, we opted to calculate the compaction gain [8] of the observed filters. This quantity is defined as [8],

$$
G_{\text {comp }} \triangleq \frac{\frac{1}{2 \pi} \int_{0}^{2 \pi}\left|F\left(e^{j \omega}\right)\right|^{2} S_{x x}\left(e^{j \omega}\right) d \omega}{\sigma_{x}^{2}}
$$

The ideal compaction filter maximizes this quantity over all magnitude squared Nyquist $(M)$ filters. A plot of the observed compaction gain as a function of the filter order parameter $N$ is shown in Fig. 5. Though the compaction gain often does come close to the optimal gain, it does not monotonically increase with $N$. Though this is counterintuitive, it is most likely due to the fact that we are constraining the desired response to have linear phase as in (21). Despite this, the observed compaction gain in many cases comes close to the ideal one. Here, the largest compaction gain observed was 2.0230 for $N=16$ compared to the ideal one of 2.0501 .

\section{CONCLUDING REMARKS}

An iterative gradient based method for designing FIR magnitude squared Nyquist filters was proposed and shown to be useful in simulations. Future research includes modifying the algorithm for compaction filter design by accounting for the arbitrary phase of the ideal compaction filter in order to obtain better compaction gains.

\section{REFERENCES}

[1] S. Akkarakaran and P. P. Vaidyanathan, "Filterbank optimization with convex objectives and the optimality of principal component forms," IEEE Trans. Signal Processing, 49(1):100-114, Jan. 2001.

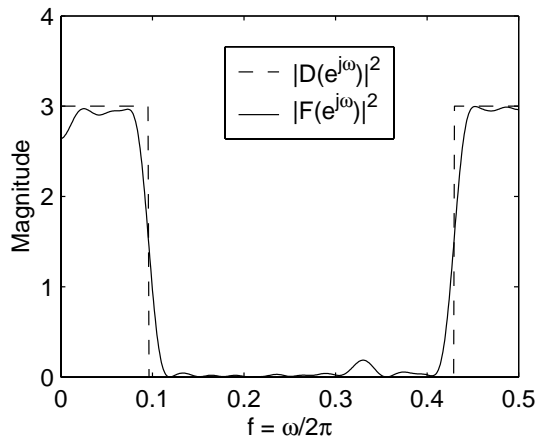

Fig. 4. Magnitude squared responses of an ideal compaction filter $D\left(e^{j \omega}\right)$ along with the designed FIR compaction filter $F\left(e^{j \omega}\right)$.

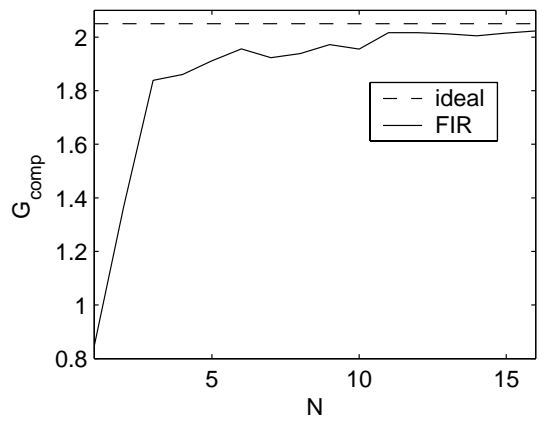

Fig. 5. Compaction gain $G_{\text {comp }}$ vs. the filter order parameter $N$.

[2] A. Kıraç and P. P. Vaidyanathan, "Theory and design of optimum FIR compaction filters," IEEE Trans. Signal Processing, 46(4):903-919, Apr. 1998.

[3] P. Moulin and M. K. Mihçak, "Theory and design of signaladapted FIR paraunitary filter banks," IEEE Trans. Signal Processing, 46(4):920-929, Apr. 1998.

[4] C. W. Therrien, Discrete Random Signals and Statistical Signal Processing, Prentice-Hall, Inc., Englewood Cliffs, NJ, 1992.

[5] M. K. Tsatsanis and G. B. Giannakis, "Principal component filter banks for optimal multiresolution analysis," IEEE Trans. Signal Processing, 43(8):1766-1777, Aug. 1995.

[6] J. Tuqan and P. P. Vaidyanathan, "A state space approach to the design of globally optimal FIR energy compaction filters," IEEE Trans. Signal Processing, 48(10):2822-2838, Oct. 2000.

[7] P. P. Vaidyanathan, Multirate Systems and Filter Banks, Prentice-Hall, Inc., Englewood Cliffs, NJ, 1993.

[8] P. P. Vaidyanathan, "Theory of optimal orthonormal subband coders," IEEE Trans. Signal Processing, 46(6):1528-1543, Jun. 1998.

[9] P. P. Vaidyanathan, T. Q. Nguyen, Z. Doğanata and T. Saramäki, "Improved technique for design of perfect reconstruction FIR QMF banks with lossless polyphase matrices," IEEE Trans. Acoust., Speech, Signal Processing, 37(7):10421056, Jul. 1989.

[10] B. Xuan and R. H. Bamberger, "FIR principal component filter banks," IEEE Trans. Signal Processing, 46(4):930-940, Apr. 1998. 